\title{
INVESTIGACIONES
}

\section{La clase de física es una extensión de la clase de matemática: percepciones de estudiantes de enseñanza media sobre la enseñanza de la física}

\author{
Physics class is an extension of the math class: \\ perceptions of high school students on the teachings of physics \\ Víctor Parra Zeltzera, Carlos Vanegas-Ortega ${ }^{b}$, \\ Denisse Bustamante González ${ }^{a}$ \\ ${ }^{a}$ Universidad Central de Chile. \\ victor.parra.zeltzer@gmail.com, denisse.bustamanteg@gmail.com \\ ${ }^{b}$ Universidad de Santiago de Chile. \\ cmariov@gmail.com
}

\section{RESUMEN}

El objetivo de esta investigación es caracterizar las percepciones que tienen los estudiantes de enseñanza media de la Región Metropolitana de Santiago de Chile sobre la matematización en las clases de física. El estudio se realizó con 1222 estudiantes de enseñanza media a quienes se les aplicó un cuestionario de escala tipo Likert de 36 preguntas, distribuidas en cuatro dimensiones: ciencias naturales, matemática, física y la clase de física. Los resultados muestran que, aunque los participantes del estudio perciben diferencias tangenciales entre la clase de física y la de matemática, consideran que la clase de física es una clase adicional de matemática (matemática aplicada). Estos resultados contribuyen a la formación inicial y continua de profesores de física porque demanda investigar sobre las mejores formas para que las clases transiten por procesos de modelización, formalización y matematización, de manera que los estudiantes puedan desarrollar visiones más cercanas a la naturaleza de la física.

Palabras clave: matematización, enseñanza de la física, aprendizaje de la física.

\begin{abstract}
The aim of this research is to characterize the perceptions that high school students from the Santiago Metropolitan Region of Chile have about mathematics in physics classes. The study was carried out with 1222 high school students, a 36-question Likert scale questionnaire was applied to them, distributed in four dimensions: natural sciences, mathematics, physics, and physics class. The results show that although the study participants perceive tangential differences between the physics and mathematics classes, they consider the physics class to be an additional class of mathematics (applied mathematics). These results put stress on practicing physics teachers and the training of physics teachers because it demands research on the best ways for classes to go through the processes of modeling, formalization and mathematization, so that students can develop closer views. to the nature of physics.
\end{abstract}

Key words: mathematization, physics teaching, physics learning. 
Estudios Pedagógicos XLVII Nº 3: 291-302, 2021

LA CLASE DE FISICA ES UNA EXTENSIÓN DE LA CLASE DE MATEMÁTICA: PERCEPCIONES DE ESTUDIANTES DE ENSEÑANZA MEDIA SOBRE LA ENSEÑANZA DE LA FÍSICA

\section{INTRODUCCIÓN}

Cuando se piensa en la enseñanza o el aprendizaje de la física, generalmente se establece una relación con la matemática, la que no siempre es clara para los estudiantes, ni para los profesores. En este sentido, existe evidencia que estos actores educativos tienen cierta tendencia a matematizar la física, confundiéndola con la mera aplicación de fórmulas y algoritmos a los conceptos y teorías físicas (Karam, 2012; Mendes y Batista, 2016; Ospina, 2011; Uhden et al., 2012; Vizcaino, 2015). Romero (2002) plantea que tanto docentes como estudiantes confunden los procesos de matematización de los fenómenos físicos con la aplicación de ejercicios matemáticos, lo que se traduce en que algunos estudiantes manipulen fórmulas sin una comprensión profunda de los conceptos físicos involucrados en ellas, y por tanto, presenten dificultades para emplear los correspondientes modelos en la realización de experimentos (Islas, 2003).

En consecuencia, la enseñanza de las ciencias, y en particular de la física, debe tener en cuenta que existe un gran número de formas y estrategias para lograr que el estudiantado comprenda los conceptos que pretenden abordar los docentes (Quibao et al., 2019). Diversas perspectivas didácticas hacen referencia a elementos que cobran importancia en la enseñanza de las ciencias, por ejemplo, la consideración de visiones más contextualizadas que apunte a aprendizajes significativos, la incorporación de la historia y la epistemología de la ciencia, así como la exploración de las ideas previas de los estudiantes (Batista da Silva et al., 2019; Mora, 2017). Lo anterior se profundiza, ya que, al revisar la literatura se evidencia que la mayoría de los docentes siguen utilizando el método tradicional de enseñanza, caracterizado por la memorización de contenidos y el escaso desarrollo de habilidades, lo que se convierte en una estrategia menos eficiente para lograr aprendizajes en los estudiantes (Contreras, 2016).

En este contexto, se han identificado algunos problemas de la enseñanza de la Física en el ámbito escolar, principalmente cuando se aborda la relación entre la Física y la Matemática, porque se considera que esta última es el lenguaje de la Física (Romero, 2002). Esta situación, es identificada en diversos países, tanto en los de habla inglesa como en Suramérica, provocando que en las clases de Física se realicen diversos ejercicios numéricos, lo que se asemejaría más a una clase de matemática (Guttersrud y Angell, 2010; Karam, 2012; Mendes y Batista, 2016; Ubaque, 2009; Uhden et al., 2012; Vizcaino, 2015). En este mismo sentido, Greca y Moreira (1998) señalan que, en el proceso de enseñanza de la física, los docentes plantean generalmente actividades y evaluaciones que se caracterizan por incluir una serie de problemas matemáticos, listas de fórmulas y definiciones, con el objetivo que estos algoritmos resulten menos complejos para los estudiantes. Sin embargo, dichos elementos no contribuyen al aprendizaje de esta ciencia y sólo aumenta la confusión entre la clase de física y la de matemática. Es así, que se ha evidenciado que los profesores de física de las escuelas tienen la tendencia a sobrecargar sus clases con ejercicios de matemática aplicada, lo que podría provocar la confusión y el cuestionamiento de los estudiantes secundarios sobre la existencia de diferencias entre la clase de matemática y la de física (Karam, 2012; Mendes y Batista, 2016; Vizcaino, 2015).

Sumado a ello, la literatura reporta que la visión que tiene el estudiantado sobre las clases de ciencia, y en particular en las clases de física, es que son instancias aburridas, difíciles, excesivamente teóricas y que implican aprender fórmulas para resolver ejercicios (Guisasola, 2004; Guttersrud y Angell, 2010; Karam, 2012; Méndez, 2014; 
Solbes, 2011; Uhden et al., 2012). Coincidentemente, Vizcaino (2015) plantea que los alumnos no cambian estas ideas, al percibir que lo que realmente les permite aprobar la asignatura son las notas obtenidas en las evaluaciones, y para ello, recurren a un alto grado de memorización, aplicación de ecuaciones y resolución mecánica de problemas rutinarios, sin preocuparse por la comprensión del fenómeno, ni por identificar por qué se está utilizando en un determinado procedimiento matemático. Lo anterior, se explica principalmente porque la participación del estudiante en la clase sigue siendo desde el rol de receptor del conocimiento que entrega el profesor (Nava, 2009). Conjuntamente, Campanario y Otero (2000) indican que los textos de estudio sugieren a los estudiantes que trabajen con fórmulas las actividades propuestas, reforzando la visión de que la física se reduce a la manipulación de un conjunto de ecuaciones.

En este contexto, dicha perspectiva genera dificultades evidentes para la comprensión de y sobre fenómenos físicos (Ayala y Garzón, 2007), dado que una parte de la información es comprendida parcialmente por el estudiante, otra es mal interpretada, y la demás, simplemente no es ni advertida, generando escasas oportunidades de aprendizaje (GómezMoliné y Sanmartí, 2000). Para generar procesos de pensamiento del estudiantado desde la mirada de la física, se deben tener en cuenta los diferentes tipos de ideas, formas de pensamiento, imágenes, ecuaciones y leyes pertenecientes a esta ciencia (Uhden et al., 2012; Ubaque, 2009). Dicho proceso no es claro que se esté produciendo en las aulas chilenas (Guisasola, 2004; Méndez, 2014; Solbes, 2011), por lo tanto, se hace necesario identificar cuál es la percepción que tienen los estudiantes de enseñanza media sobre la clase de física. Es por ello, que se indagó la siguiente pregunta de investigación: ¿Cómo ha influido la matematización en la forma como perciben la física los estudiantes de enseñanza media de algunos colegios de Santiago de Chile?

En este marco, el objetivo de esta investigación es caracterizar las percepciones que tienen los estudiantes de enseñanza media de algunos colegios de Santiago de Chile sobre la matematización en las clases de física. Se ha planteado una hipótesis explicativa, la que señala que los estudiantes de enseñanza media perciben que las clases de física se han transformado en clases de matemática aplicada, por lo que, al resolver ejercicios numéricos piensan que han aprendido física.

\section{MARCO TEÓRICO}

Entendiendo que este estudio aborda la matematización de la clase de física, se debe abordar en primer lugar, la relación entre la Física y la Matemática. En este sentido, es necesario tener claridad sobre el rol que tiene la Matemática en la Física, desde el entendido que toda magnitud matemática no es una magnitud física. Existe una relación entre la física y la matemática, donde el físico le asigna un determinado significado a las variables que utilizan que no es el mismo significado que le asigna el matemático, ya que en la física cada variable entrega información adicional a su valor numérico (Paty, 2006). En coherencia con lo anterior, Redish (2006) indica que, en la Física, los símbolos no son arbitrarios, pero son elegidos para activar una asociación mental, en particular, con alguna cantidad Física o medición. No obstante, dicha asociación mental no es matemática, lo que se busca es que la expresión tenga sentido para aquellos que la leen, además de evocar a algún fenómeno de la realidad. 
Estudios Pedagógicos XLVII N 3: 291-302, 2021

LA CLASE DE FÍSICA ES UNA EXTENSIÓN DE LA CLASE DE MATEMÁTICA: PERCEPCIONES DE ESTUDIANTES DE ENSEÑANZA MEDIA SOBRE LA ENSEÑANZA DE LA FÍSICA

En segundo término, se debe considerar que los conceptos físicos poseen características que pueden presentarse bajo la forma de magnitudes que tiene una expresión matemática, y a la vez, las leyes que rigen un fenómeno físico se formulan bajo la forma de ecuaciones que relacionan estas magnitudes entre sí (Paty, 2006). Sin embargo, Vizcaino (2015) indica que la relación entre la Física y la Matemática va más allá del mero uso de cálculos numéricos y le da a la Matemática el estatus de lenguaje que le permite al físico explorar de mejor manera las leyes que rigen un fenómeno.

Lo anterior implica que, en las clases de Matemática se resuelven ejercicios que son numéricos, sin embargo, en la clase de Física, además de resolver problemas y ejercicios, se le debe dar importancia a la comprensión de los conceptos. En esta línea, es necesario entender que la física y la matemática son ciencias diferentes que presentan una relación estrecha, y por tanto, la literatura señala que esto hace mas imperiosa la necesidad de diferenciarlas durante los procesos de enseñanza (Karam, 2012; Malagón, 2013; Uhden et al., 2012).

Si bien existen varias maneras de concebir los procesos de enseñanza de las ciencias, y en específico los de la Física (Becerra-Labra y Gras-Marti, 2010; Furió-Más y FurióGómez, 2012), este estudio adopta la propuesta de Ayala y Grazón (2007), quienes plantean la enseñanza de la física como un proceso que conlleva transitar por tres momentos: modelizar, formalizar y matematizar.

Modelizar corresponde al momento en que se genera una imagen mental sobre el concepto que se está intentando explicar. Es así, que los modelos constituyen el núcleo del conocimiento científico y la modelización es la actividad sistemática a partir de la cual se puede construir y emplear este conocimiento; a su vez, en esta parte del proceso tanto el docente como los estudiantes, ponen en juego sus propios modelos sobre el fenómeno físico (Romero, 2002). Ahora, al considerar una situación cualquiera de aprendizaje en el ámbito de la física se puede decir que, al comenzar a trabajar con un concepto se debe intentar que el estudiante sea capaz de plantear su propio modelo y eso implica establecer una imagen mental que le permita explicarlo (en lenguaje cotidiano o socialmente compartido), es decir, que las leyes de la Física aún en el nivel más bajo, no se aplican directamente al hecho físico sino a los modelos que sobre ellos construyen los sujetos (Colombo y Pesa, 2008). En consecuencia, la modelización se relaciona directamente con la generación de modelos mentales (Galagovsky y Aduriz-Bravo, 2001). Para este trabajo se considera la modelización como el establecimiento de relaciones semánticas entre la teoría y los fenómenos u objetos (Greca y Moreira, 1998).

Formalizar concierne al segundo momento donde se le asignan las estructuras del lenguaje científico a los hechos físicos que se están estudiando. En este sentido, Ayala y Garzón (2007) consideran la formalización como un proceso natural del pensamiento o de todo proceso cognitivo en la medida en que el lenguaje común, es en sí mismo, un proceso de formalización. En efecto, cuando se enseña un concepto nuevo, lo primero que se debe hacer es indicarle al estudiante en qué espacio le es útil, para qué le sirve y qué le permitirá explicar; todo ello con el propósito de que pueda construir un discurso articulado para referirse al fenómeno en particular. Entonces, este proceso resulta de suma importancia a la hora de la construcción del aprendizaje por parte de los estudiantes, ya que es en este momento cuando los estudiantes se apropian de conceptos y procedimientos vinculados al hecho estudiado, demostrando que son capaces de hacer suyo el lenguaje científico que se les ha enseñado (Malagón, 2013). 
Matematizar es el momento donde se plantea la manipulación de los símbolos, es decir, se reconoce una estructura formal utilizando una simbología matemática que contribuye a la explicación del fenómeno físico (Ayala y Garzón, 2007). En esta línea, Romero (2002) indica que matematizar un fenómeno físico no consiste en sobreponer un aparato matemático al fenómeno; sino de construir las magnitudes, relaciones y procedimientos apropiados para representarlo y cuantificarlo. Para que la aplicación de la estructura matemática a un fenómeno físico tenga sentido, la matematización debe estar supeditada a la formalización que se realice del fenómeno en estudio (Paty, 2006). En resumen, matematizar conlleva un proceso de pensamiento que es mucho más que solo resolver problemas donde se aplican procedimientos matemáticos, implica un proceso mental necesario para aprender física.

Con base en los tres momentos descritos, es importante destacar que los profesores de física deben comprender cómo las relaciones entre la Física y la Matemática han sido desarrolladas en la construcción del conocimiento científico. Esto realza la importancia de prestar atención al uso del lenguaje, ya que en el entendido de que la matemática son parte del lenguaje de la física, también desde ese lenguaje se realiza la construcción de la ciencia escolar. En lo especifico, se debe diferenciar entre un lenguaje puramente matemático y un lenguaje matemático asociado a la representación de los fenómenos físicos, debido a que, en la Matemática, el lenguaje tiene un carácter de abstracción diferente al de la Física (Vizcaino, 2015).

\section{METODOLOGÍA}

Esta investigación utilizó una metodología cuantitativa, utilizado un diseño transversal de tipo exploratorio-descriptivo, dado que permite familiarizarse con un aspecto desconocido, poco desarrollado y novedoso para el campo de estudio, asociado a identificar las percepciones que tienen los estudiantes de Santiago de Chile sobre las clases de física (López et al., 2018). El estudio se realizó con 1.222 estudiantes de enseñanza media de la Región Metropolitana de Santiago de Chile, quienes aceptaron voluntariamente responder un cuestionario, distribuido masivamente a través de correo electrónico y redes sociales. La aplicación on-line del instrumento evitó sesgos con respecto al tipo de institución, edad, sexo o lugar de residencia.

Para caracterizar la percepción que tienen algunos estudiantes de enseñanza media sobre la matematización en las clases de física, se les aplicó un cuestionario de escala tipo Likert que tiene posibilidades de respuesta desde el muy en desacuerdo al muy de acuerdo. Como muestra la tabla 1, el cuestionario consta de 36 preguntas distribuidas en cuatro dimensiones: ciencias naturales, matemática, física y la clase de física. Para garantizar el rigor científico (Klees, 2017), el instrumento fue piloteado con 116 estudiantes de enseñanza media de la Región Metropolitana de Santiago de Chile, además, para verificar la validez interna del instrumento se calculó el alfa de Cronbach $(\alpha=0.89)$. 
Estudios Pedagógicos XLVII Nº 3: 291-302, 2021

LA CLASE DE FISICA ES UNA EXTENSIÓN DE LA CLASE DE MATEMÁTICA: PERCEPCIONES DE ESTUDIANTES

DE ENSEÑANZA MEDIA SOBRE LA ENSEÑANZA DE LA FÍSICA

Tabla 1. Estructura del cuestionario de percepciones aplicado a los estudiantes

\begin{tabular}{|c|l|c|}
\hline Dimensión & \multicolumn{1}{|c|}{ Objhetivo Específico } & Preguntas \\
\hline $\begin{array}{c}\text { Ciencias } \\
\text { Naturales }\end{array}$ & $\begin{array}{l}\text { Identificar las percepciones sobre las ciencias naturales de } \\
\text { algunos estudiantes de enseñanza media de la Región } \\
\text { Metropolitana de Santiago. }\end{array}$ & $1-9$ \\
\hline Matemática & $\begin{array}{l}\text { Reconocer las percepciones sobre la matemática de algunos } \\
\text { estudiantes de enseñanza media de la Región Metropolitana de } \\
\text { Santiago. }\end{array}$ & $10-18$ \\
\hline Física & $\begin{array}{l}\text { Determinar las percepciones sobre la física de algunos estudiantes } \\
\text { de enseñanza media de la Región Metropolitana de Santiago. }\end{array}$ & $4,19-29$ \\
\hline Clase de Física & $\begin{array}{l}\text { Identificar las percepciones sobre la matematización de las clases } \\
\text { de física de algunos estudiantes de enseñanza media de la Región } \\
\text { Metropolitana de Santiago. }\end{array}$ & $\begin{array}{c}15,26, \\
30-36\end{array}$ \\
\hline
\end{tabular}

\section{RESULTADOS}

La primera dimensión del cuestionario hace referencia a la percepción que tienen los estudiantes sobre la enseñanza de las ciencias naturales. Los estudiantes indicaron estar mayoritariamente de acuerdo (35\% y $40 \%$ entre muy de acuerdo y de acuerdo, respectivamente) con las aseveraciones que hacían referencia a las ciencias naturales y su aprendizaje, en ese sentido la mayoría tiene una percepción positiva de las ciencias naturales o su aprendizaje. La mayoría ( $26 \%$ muy de acuerdo y $51 \%$ de acuerdo) cree que las ciencias que aprende en el colegio serán útiles para su vida futura. Esta última idea se contrapone con la percepción que tienen los estudiantes sobre las clases de ciencias naturales, donde la mayoría piensa que las clases son aburridas ( $\mathrm{DE}=0,75)$, como muestra en la figura 1 .

Figura 1. Percepción de estudiantes de enseñanza media sobre las clases de ciencias naturales.

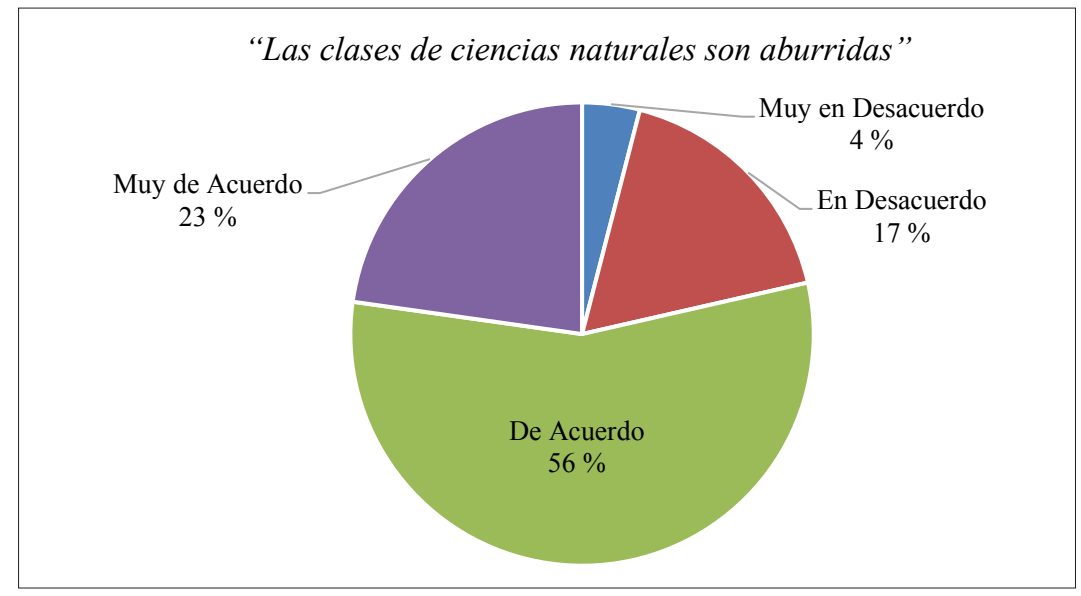


En este sentido, resulta preocupante la visión que tienen los estudiantes sobre la interrelación entre sus dos visiones, aunque se ha dicho que creen que es importante saber ciencias, la mayoría ( $18 \%$ muy de acuerdo y $40 \%$ de acuerdo), creen que la forma en la que se hacen las clases no les llama la atención $(\mathrm{DE}=0,88)$ (Figura 2). Este resultado ratifica la demanda de distintos investigadores en didáctica de las ciencias para seguir avanzando en la búsqueda de la transformación de los ambientes de aprendizaje, a partir de las características de los contextos educativos y de los intereses de los estudiantes (Batista da Silva et al., 2019; Cabrera et al., 2010; Contreras, 2016; Guttersrud y Angell, 2010; Karam, 2012; Méndez, 2014; Mora, 2017; Quibao et al., 2019; Solbes, 2011; Uhden et al., 2012).

Figura 2. Percepción de estudiantes de enseñanza media sobre las clases de ciencias naturales.

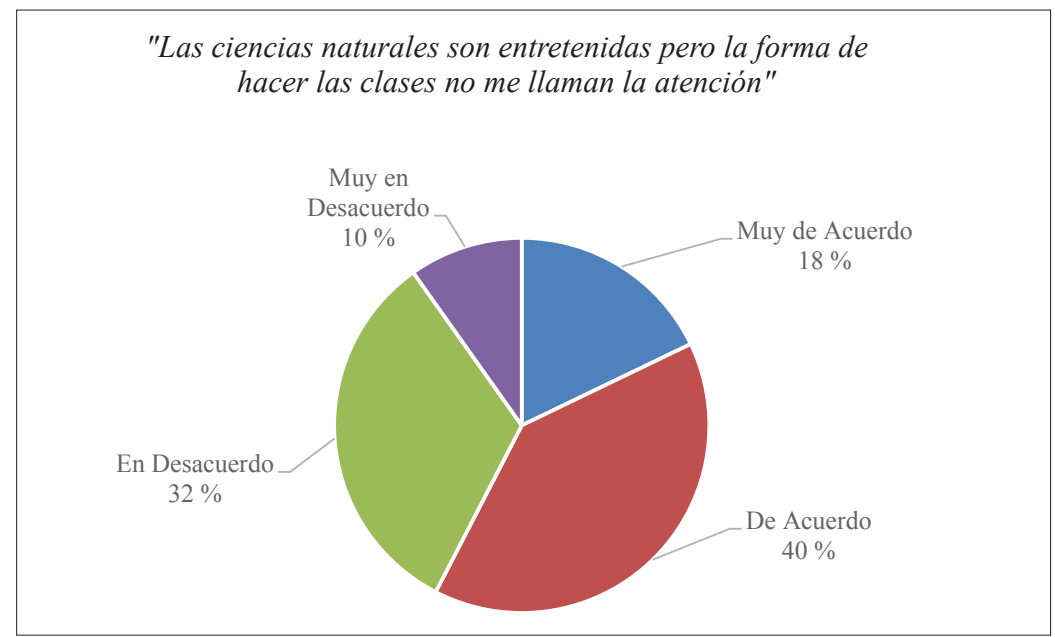

Figura 3. Percepción de estudiantes de enseñanza media sobre las matemáticas.

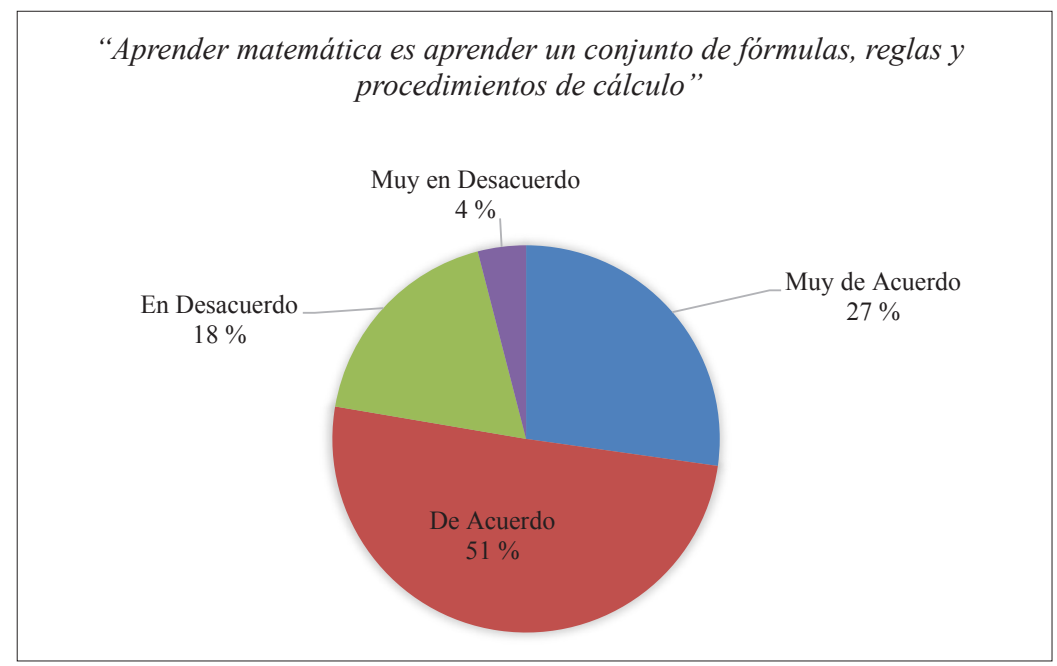


La segunda dimensión del cuestionario hace referencia a la percepción que tienen los estudiantes de la Región Metropolitana de Santiago sobre la enseñanza de la matemática. En esta dimensión se nota que la percepción que tienen es positiva, ya que el 70\% indica estar de acuerdo o muy de acuerdo cuando se les pregunta si les gusta la matemática o, por el contrario, el $40 \%$ contestó estar de acuerdo o muy de acuerdo cuando se les preguntó si encontraban difícil la matemática. Además, sobre el aprendizaje de la matemática, la mayoría indicó que era importante aprenderla (66\% muy de acuerdo y $30 \%$ de acuerdo). Otro elemento significativo de los resultados de esta dimensión es que la mayoría de los estudiantes $(78 \%)$ indicó que el aprendizaje de la matemática consistía en aprender un conjunto de fórmulas, reglas y procedimientos de cálculo $(\mathrm{DE}=0,79)$, tal y como muestra la figura 3. En esta misma dimensión se puede señalar que un número importante de estudiantes (41\%) declaran que se aburren en clases de matemática y que les cuesta trabajo responder los ejercicios de cálculo.

La tercera dimensión del cuestionario hace referencia a la percepción que tienen los estudiantes sobre la enseñanza de la física. En esta dimensión es importante resaltar que la mayoría (66\%) indica que le gusta la física pero, aproximadamente el mismo porcentaje (62\%) indica que le parece difícil; este dato puede estar relacionado con el hecho de que prácticamente la mitad de los estudiantes (48\%) indica que se aburre en las clases de física o con el cómo se hacen dichas clases, ya que el $83 \%$ indica estar de acuerdo o muy de acuerdo con que la comprendería mejor si se le explicara con situaciones cotidianas.

La mayoría de los estudiantes manifestaron que la física es matemática aplicada (DE $=0,49)(25 \%$ muy de acuerdo y $54 \%$ de acuerdo), como se ilustra en la figura 4 . Este resultado se condice con lo que plantean Ayala y Garzón (2007) cuando señalan que en la enseñanza de la física es muy común que se aborde la conceptualización (si es que se aborda) separada de la matematización.

Figura 4. Percepción de estudiantes de enseñanza media sobre la relación entre física y matemáticas.

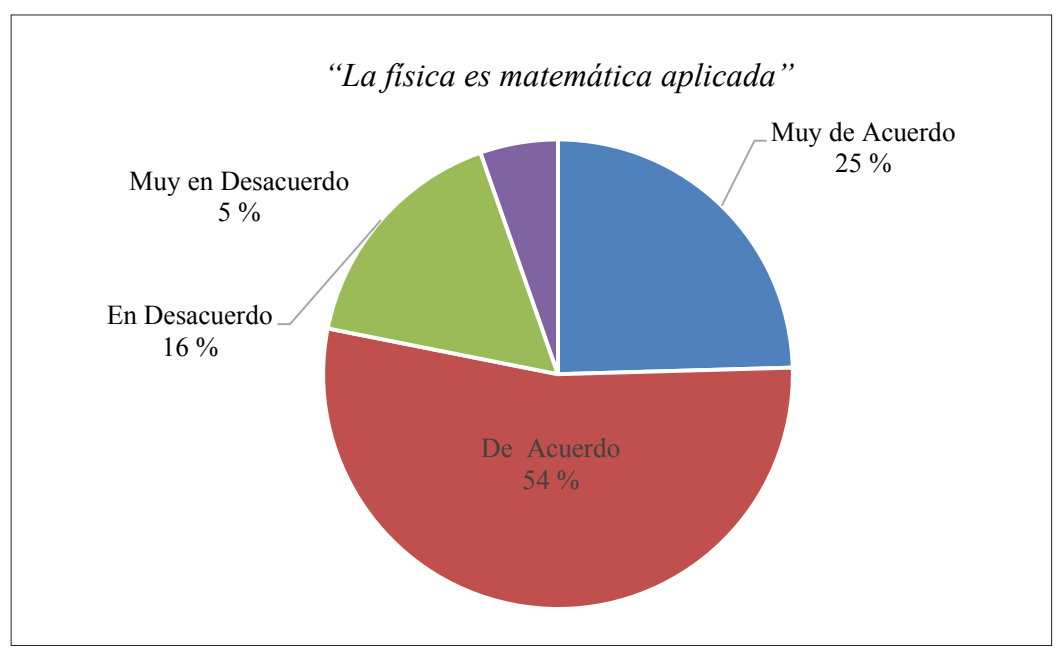


En coherencia con Ospina (2011), la mayoría de los estudiantes contestaron que aprender física es aprender un conjunto de fórmulas, reglas y procedimientos de cálculo ( $\mathrm{DE}=0,87$ ), como se muestra en la figura 5. No obstante, el 54\% de los estudiantes encuestados advierte que no está de acuerdo con la percepción de que las clases de física siempre deban tener ejercicios numéricos ( $\mathrm{DE}=0,77$ ) (ver figura 6), por lo que se puede señalar que los alumnos logran identificar diferencias entre los procesos de aprendizaje de ambas asignaturas.

Figura 5. Percepción de estudiantes de enseñanza media sobre el aprendizaje de la física.

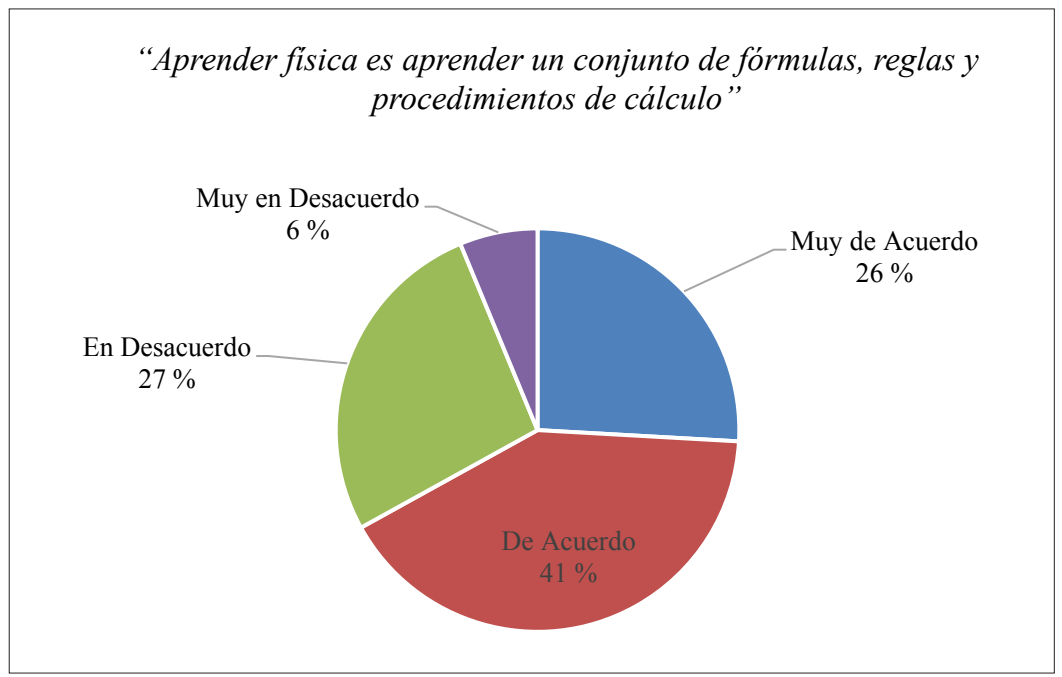

Figura 6. Percepción de estudiantes de enseñanza media sobre el aprendizaje de la física.

"Las clases de física siempre deben tener ejercicios numéricos"

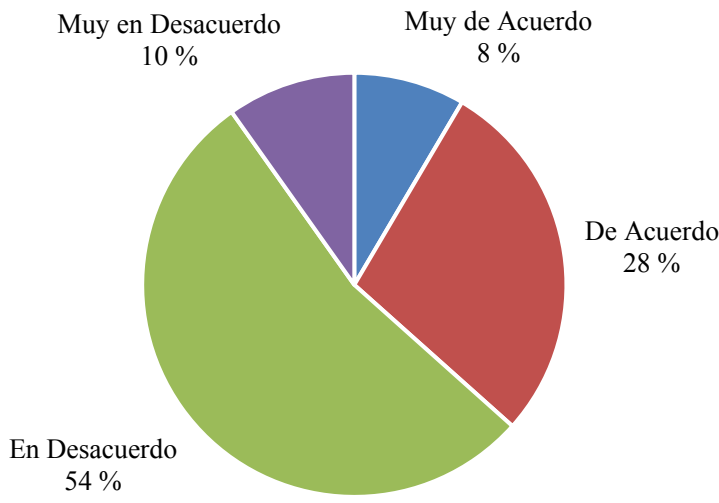


Estudios Pedagógicos XLVII N 3: 291-302, 2021

LA CLASE DE FISICA ES UNA EXTENSIÓN DE LA CLASE DE MATEMÁTICA: PERCEPCIONES DE ESTUDIANTES DE ENSEÑANZA MEDIA SOBRE LA ENSEÑANZA DE LA FÍSICA

Estos resultados deben hacer reflexionar al profesorado (en ejercicio y en formación), así como a los formadores de profesores, sobre qué y cómo se está enseñando física a los estudiantes, ya que, si solamente se considera que hay que resolver ejercicios para aprender física, es probable que se pierda de vista el fenómeno físico sobre el cual se está trabajando. Esto se condice con lo que plantea Ospina (2011) cuando indica que, en docentes y alumnos, se evidencia una tendencia a matematizar la física confundiéndola con la mera aplicación de fórmulas y algoritmos a los conceptos y las teorías físicas.

Por otro lado, la cuarta dimensión del cuestionario hace referencia a la relación que establecen los estudiantes entre la física y la matemática. En esta dimensión es notable que el $80 \%$ de los estudiantes declaran ser capaces de hacer diferencias entre estas; sin embargo, la mayoría señala estar de acuerdo con que la física siempre debe tener números, que el objetivo de la clase de física es resolver ejercicios o, que lo importante en un problema de física es el resultado numérico. Esta contradicción es explicable en función de los resultados: en el imaginario general de los estudiantes, la física y la matemática son ciencias que poseen diferencias, sin embargo, de acuerdo con las aproximaciones que han tenido con la física en el sistema escolar, más del 70\% de ellos consideran que esta se aprende si contestan bien los ejercicios numéricos.

\section{CONCLUSIONES}

En consecuencia, las ideas que tienen los estudiantes de educación media de la Región Metropolitana de Chile sobre la enseñanza de las ciencias naturales es que dichas ciencias son tediosas y monótonas, lo que repercute directamente en la forma como perciben las clases de física, y por tanto, la mayoría manifiesta sentirse aburrida en esta asignatura. A su vez, se puede advertir que, si bien los estudiantes reconocen la importancia de la matemática para su proceso educativo, ellos consideran que esta asignatura se basa en aprender sólo formulas, reglas y procedimientos de cálculo, teniendo la percepción de que dichas clases son tediosas.

Se puede concluir que los estudiantes creen haber aprendido física cuando resuelven los ejercicios que el profesor propone en clases, lo que los lleva a aseverar que la física es matemática aplicada. Es posible afirmar que los estudiantes encuestados perciben que la enseñanza de la física se ha matematizado; es decir, se omiten los procesos históricos y epistemológicos que le entregan una naturaleza propia al estudio y comprensión de los fenómenos físicos (Ayala y Garzón, 2007; Malagón, 2013; Romero, 2002), lo que invisibiliza las diferencias entre el lenguaje puramente matemático y un lenguaje matemático asociado a la representación de estos fenómenos (Vizcaino, 2015).

En conclusión, aunque los estudiantes de enseñanza media de la Región Metropolitana de Santiago de Chile perciben diferencias entre la clase de física y la de matemática, consideran que la física es matemática aplicada, por lo que, aprenden física si resuelven los ejercicios numéricos propuestos por el profesor. Estos resultados ponen en tensión al profesorado de física en ejercicio y a la formación de profesores de física, en especial porque invita a la reflexión sobre cómo los docentes conciben el proceso de enseñanza de esta disciplina (Furió-Más y Furió-Gómez, 2012; Islas, 2003). Desde la perspectiva teórica de este estudio (Ayala y Garzón, 2007), la tensión se centra en indagar por las mejores formas de transitar por la modelización, la formalización y la matematización; así como 
reconocer diversidad de estrategias y recursos que permitan a los estudiantes desarrollar visiones más cercanas a la naturaleza de la física.

Dado que los estudiantes de enseñanza media perciben que la clase de física es una clase adicional de matemática, las investigaciones chilenas en didáctica de esta materia se encuentran ante el desafío de responder las siguientes preguntas: ¿cómo transformar los ambientes de aprendizajes de las clases de física para que los estudiantes transiten por la modelización, formalización y matematización?, ¿los procesos evaluativos de la clase de física privilegian la matematización sobre la modelización y la formalización?, ¿cuáles son las visiones epistemológicas sobre la física que tiene el profesorado del sistema escolar y cómo se relacionan con sus prácticas de enseñanza?, ¿qué lugar ocupa la matematización de los fenómenos físicos en los programas de formación inicial de profesores?, ¿cuáles son las comprensiones sobre la enseñanza de la física que están adquiriendo los docentes en formación inicial?, ¿comprenden los futuros profesores las diferencias entre modelizar, formalizar y matematizar?, ¿cuentan el profesorado con la formación disciplinar, didáctica y pedagógica para diseñar ambientes de aprendizajes que le posibilite a sus estudiantes transitar por la modelización, formalización y matematización?, ¿cómo influye la presencia de la matematización de la física durante la formación inicial de profesores en las formas de comprender la enseñanza de la física?

\section{REFERENCIAS BIBLIOGRÁFICAS}

Ayala, M. M. y Garzón, M. (2007). Consideraciones sobre la formalización y matematización de los fenómenos físicos. Praxis filosófica, 25, 39-54.

Batista da Silva, J., Leite Sales, G. y Braga de Castro, J. (2019). Gamificação como estratégia de aprendizagem ativa no ensino de Física. Revista Brasileira de Ensino de Física, 41(4).

Becerra-Labra, C. y Gras-Martí, A. (2010). Effects upon the ability of "paper and pencil" problem solving of physics teaching and learning with a problem-based structure. Revista Brasileira de Ensino de Física, 32(2), 1-11.

Cabrera, E., González, J., Montenegro, E. y Nettle, A. (2010). Una didáctica del saber: un camino hacia la optimización de las transposiciones didácticas. Estudios pedagógicos, 36(2), 51-61.

Campanario, J. M. y Otero, J. (2000). Más allá de las ideas previas como dificultades de aprendizaje: Las pautas de pensamiento, las concepciones epistemológicas y las estrategias metacognitivas de los alumnos de ciencias. Enseñanza de las ciencias, 18(2), 35-48.

Colombo, L. y Pesa, M. A. (2008). La evolución de los significados de los conceptos científicos en relación con la estructura cognitiva de los estudiantes. Ciência \& Educação, 14(3), 365-380.

Contreras, S. A. (2016). Pensamiento Pedagógico en la Enseñanza de las Ciencias.Análisis de las Creencias Curriculares y sus Implicancias para la Formación de Profesores de Enseñanza Media. Formación Universitaria, 9(1), 15-24.

Furió-Más, C. y Furió-Gómez, C. (2012). Aprofundando a educação científica: aspectos epistemológicos e metodológicos a se considerar no ensino. Educar em Revista, 44, 37-57.

Galagovsky, L. R. y Adúriz-Bravo, A. (2001). Modelos y analogías en la enseñanza de las ciencias naturales. El concepto de modelo didáctico analógico. Enseñanza de las Ciencias, 19(2), 231242.

Gómez-Moliné, M. y Sanmartí, N. (2000). Reflexiones sobre el lenguaje de la ciencia y el aprendizaje. Educación Química, 11(2), 266-273.

Greca, I. y Moreira, M. (1998). Modelos mentales, modelos conceptuales y modelización. Enseñanza de la física, 15(2), 107-120. 
Estudios Pedagógicos XLVII N 3: 291-302, 2021

LA CLASE DE FISICA ES UNA EXTENSIÓN DE LA CLASE DE MATEMÁTICA: PERCEPCIONES DE ESTUDIANTES

DE ENSEÑANZA MEDIA SOBRE LA ENSEÑANZA DE LA FÍSICA

Guisasola, J. (2004). ¿Puede ayudar la investigación en enseñanza de la Física a mejorar su docencia en la universidad? Revista Brasileira de Ensino de Física, 26(3), 197-202.

Guttersrud, Ø. y Angell, C. (2010). Mathematics in physics: Upper secondary physics students' competency to describe phenomena applying mathematical and graphical representations. En: girep-icpe mptl Conference. Reims France.

Islas, S. M. (2003). ¿Qué rol asignan los profesores de física de nivel medio a los modelos científicos y a las actividades de modelado? Enseñanza de las ciencias , Número Extra, 57-66.

Karam, R. A. S. (2012). Estruturação matemática do pensamento físico no ensino: uma ferramenta teórica para analisar abordagens didáticas. Tesis de doctorado. Universidade de São Paulo.

Klees, S. J. (2017). Quantitative Methods in Comparative Education and Other Disciplines: are they valid?. Educação \& Realidade, 42(3), 841-858.

López, N., Erwin, C., Binder, M. y Chavez, M. J. (2018). Making the invisible visible: Advancing quantitative methods in higher education using critical race theory and intersectionality. Race Ethnicity and Education, 21(2), 180-207.

Malagón, F. S. (2013). La actividad experimental: Construcción de fenomenologías y procesos de formalización. Praxis Filosófica, 36, 119-138.

Méndez, D. (2014). Influencia de la inteligencia y la metodología de enseñanza en la resolución de problemas de Física. Perfiles Educativos, 36(146), 30-44.

Mendes, G. H. G. I. y Batista, I. D. L. (2016). Matematização e ensino de Física: uma discussão de noções docentes. Ciência \& Educação (Bauru), 22(3), 757-771.

Mora, C. (2017). Cambiando paradigmas en la enseñanza de las ciencias: consideraciones sobre el aprendizaje activo de la física. Revista Areté. Revista Amazônica de Ensino de Ciências, 1(1), 24-32.

Nava, M. (2009). Referentes teóricos de una instrucción orientada a la construcción de conceptos científicos en física. Revista de la Facultad de Ingeniería U.C.V, 24(4), 98-111.

Ospina, A. (2011). A propósito de la formalización de los fenómenos físicos: Un análisis desde la perspectiva newtoniana. Revista Científica, Extra, 215-224.

Paty, M. (2006). Einstein y el rol de las matemáticas en la física. Praxis Filosófica, 22, 5-28.

Quibao, M. P., Silva, A. C., Almeida, N. S. D., Silva, R. M. A. A., Muniz, S. R. y Paiva, F. F. (2019). Investigando a compreensão conceitual em física de alunos de graduação em cursos de ciências, engenharias e matemática. Revista Brasileira de Ensino de Física, 41(2).

Redish, E. (2006). Changing student ways of knowing: What should our students learn in a physics class. Proceedings of World View on Physics Education 2005: Focusing on Change (pp. 1-13). University of Delhi.

Romero, Á. R. (2002). La formalización de los conceptos físicos. El caso de la velocidad instantánea. Revista Educación y Pedagogía, 15(36), 57-67.

Solbes, J. (2011). ¿Por qué disminuye el alumnado de ciencias? Alambique Didáctica de las Ciencias Experimentales, (67), 53-61.

Ubaque, K. Y. (2009). Sobre el significado de la didáctica de la física. Gondola, 4(1), 19-24.

Uhden, O., Karam, R., Pietrocola, M. y Pospiech, G. (2012). Modelling mathematical reasoning in physics education. Science \& Education 21(4), 485-506.

Vizcaino, D. F. (2015). Diferencias trascendentales entre matematización de la física y matematización para la enseñanza de la física. Tecné Episteme y Didaxis, 16(1), 95-111. 